\title{
Deutsche Unternehmer und ihre Arbeiterkolonien im 19. und frühen 20. Jahrhundert
}

STEFFEN KRÄMER

Schon seit dem letzten Viertel des 18. Jahrhunderts entstanden vorwiegend in England kollektive Wohngebäude in unmittelbarer Nähe größerer Fabrikanlagen für die werktätigen Arbeiter und ihre Familien. Die Bauträger waren in der Regel die Unternehmer selbst, die dadurch das notwendige Potential an Arbeitern an die Fabrik binden konnten. Aus diesen eher bescheidenen Anfängen des von privaten Unternehmern getragenen kollektiven Wohnungsbaus entwickelten sich in England im Verlauf des 19. Jahrhunderts große Arbeitersiedlungen oder Industriedörfer in räumlicher Nachbarschaft zu den Fabriken mit teilweise mehreren tausend Einwohnern.

Ein berühmtes Beispiel ist die Siedlung Saltaire des Textilfabrikanten Sir Titus Salt, der seine Fabrik mitsamt dem angegliederten Arbeiterdorf mit über 4.000 Einwohnern ab 1851 auf dem Land in der Nähe der mittelenglischen Stadt Bradford errichten ließ (Posener 1968:22-27; Reinborn 1996:35); (Abb. 1). Was diese englischen Unternehmersiedlungen aus dem 19. Jahrhundert so außerordentlich interessant macht, ist die in der Regel bewusst kalkulierte Verbindung von wirtschaftlichen Erwägungen mit sozialreformerischen Ideen. Nur kurze Zeit nach ihren englischen Vorläufern erkannten auch deutsche Unternehmer den hohen Wert solcher Wohnsiedlungen für ihre Werksarbeiter und deren Familien. 
Abb. 1: Saltaire bei Bradford, Arbeitersiedlung der Textilfabrik, ab 1851, Grundriss der Gesamtanlage

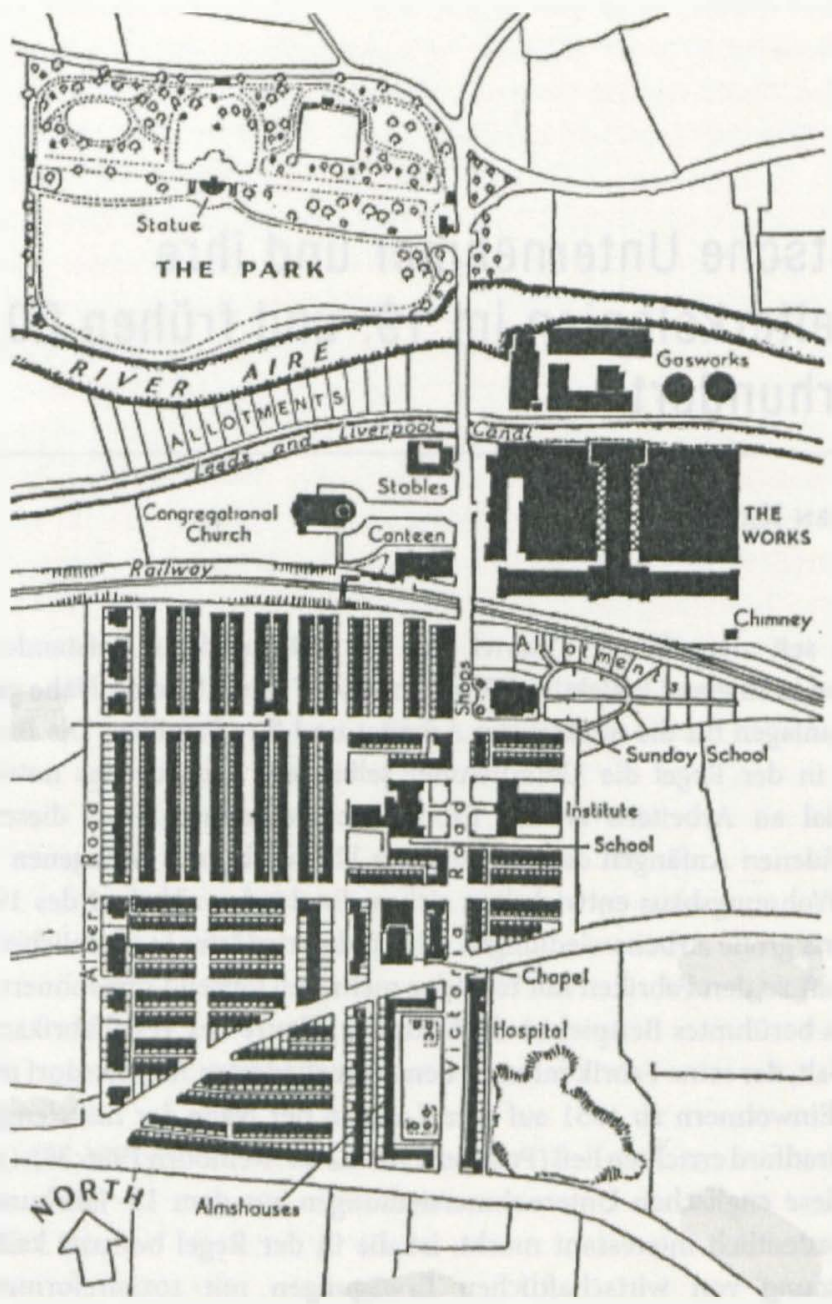

\section{Die Arbeiterkolonien der Gussstahlfabrik Krupp IN ESSEN}

Die zweifellos bekanntesten Beispiele im Verlauf des 19. und zu Beginn des 20. Jahrhunderts stellen die so genannten „Arbeiterkolonien“ der Gussstahlfabrik Krupp in Essen und Umgebung dar (Günter 1970:128-174; Schlandt 1970:95-111; Kastorff-Viehmann 1981:153-158; Kieß 1991:373-392); (Abb. 2). Zwar gab es bereits vor den Krupp-Kolonien eine nicht geringe Anzahl von paternalistischen Arbeitersiedlungen vor allem auch im Ruhrgebiet, doch war die Firma Krupp das erste deutsche Unternehmen, das seit den frühen 1860er 
Jahren Werksunterkünfte in einem erstaunlich umfassenden Maße zu errichten begann.

Abb. 2: Essen, Arbeiterkolonien der Gussstahlfabrik Krupp, 1902, Übersichtskarte

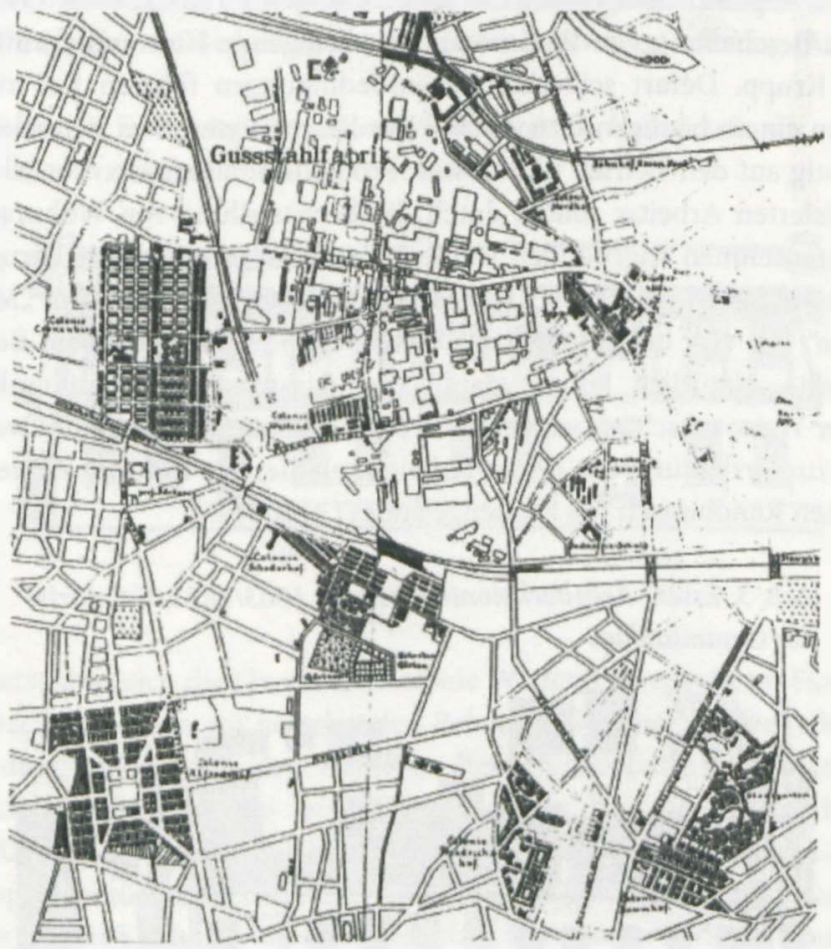

1874, also ungefähr zehn Jahre nach dem Beginn der Kruppschen Wohnbautätigkeit, waren bereits 2.358 Arbeiterwohnungen größtenteils in direkter Nähe zum Fabrikgelände fertiggestellt (Wohlfahrtseinrichtungen I 1902:4-5). In der englischen Arbeitersiedlung Saltaire wurden in einem vergleichbaren Zeit raum dagegen nur etwa 850 Wohneinheiten errichtet (Posener 1968:26).

Allerdings war es nicht die damalige Firmenleitung, sondern der Fabrikbesitzer Alfred Krupp (1812-1887) persönlich, der dieses ehrgeizige Siedlungsprogramm bereits Ende der 1850er Jahre zu initiieren begann und es bis zu seinem Tod 1887 über mehrere Jahrzehnte hinweg systematisch vorantrieb. Die historischen Ursachen und Motive, die sich dahinter verbergen, sind vielschichtig und im Vergleich zu den etwa zeitgleichen Wohnbaukonzepten der englischen Unternehmer durchaus auch ambivalenter. Wenn man sich mit diesem ambitionierten Siedlungsprogramm näher beschäftigen möchte, ergibt sich aber das grundsätzliche Problem, dass alle Arbeiterkolonien, die unter Alfred Krupp errichtet wurden, heute nicht mehr erhalten sind. Schon in den 1930er Jahren wurden die meisten fabriknahen Siedlungen abgerissen, 
weil man die Produktionsstätten auf dem Werksgelände vergrößerte (Essen und Umgebung 1938:42). Die folgende Analyse beruht demnach ausschließlich auf historischem Abbildungs- und Planmaterial.

Vor dem Hintergrund der desolaten Wohnungsmisere in Essen, die in den 1860er Jahren mit etwa 23 Bewohnern pro Arbeiterhaus ihren dramatischen Höhepunkt fand (Wohlfahrtseinrichtungen I 1902:3; Kieß 1991:374), war die Beschaffung von Wohnraum eine dringende Notwendigkeit für die Firma Krupp. Derart schlechte Lebensbedingungen führten fast zwangsläufig zu einem häufigen Ortswechsel der Beschäftigten, was sich wiederum ungünstig auf den Betrieb und dessen Produktion auswirkte. Vor allem die spezialisierten Arbeiter sollten durch die Bereitstellung von Wohnraum an das Unternehmen angebunden werden. Demzufolge begann die Kruppsche Wohnbautätigkeit 1861 mit dem Bau von zwei so genannten „Meisterhäusern" mit vier beziehungsweise sechs Wohnungen für höhere Betriebsangestellte. Natürlich konnte damit die katastrophale Wohnungslage in keinster Weise entschärft werden, und so entschloss man sich nur zwei Jahre später zur Errichtung der ersten Arbeiterkolonie, der Kolonie Westend, im südlichen Randbereich des Firmengeländes (Abb. 3).

Abb. 3: Essen, Arbeiterkolonie Westend, 1863/1871, Grundriss der Gesamtanlage

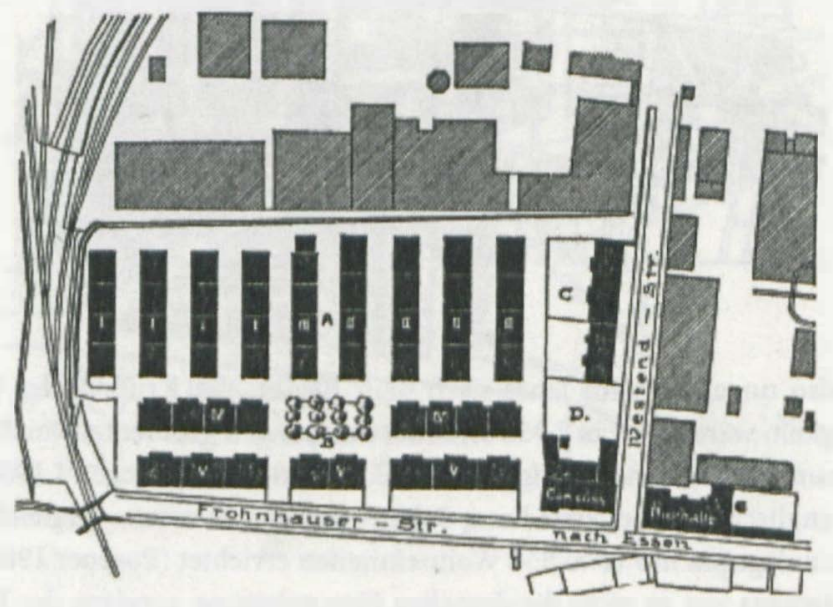

Schon bei dieser ersten Arbeiterkolonie war die Lage in unmittelbarer Nähe zu den Produktionsstätten außerordentlich wichtig. Die geringe Entfernung sollte garantieren, dass der Arbeiter über Mittag nach Hause ging, um danach sofort wieder im Betrieb zu erscheinen. Sein gesamter Tagesablauf war somit ausschließlich auf die Fabrik ausgerichtet. Für die Planung der Arbeiterkolonie wurde bereits ein firmeneigenes Baubüro eingerichtet, das zunächst neun parallel angeordnete Häuserzeilen mit insgesamt 136 Wohnungen errichtete. In der Regel hatten diese Wohnungen zu Anfang nur 35 Quadratmeter 
und waren in zwei Räume aufgeteilt: in eine Küche, die gleichzeitig Wohnraum war, und in ein Schlafzimmer. Noch während der Bauzeit erhöhte man allerdings zum Teil die Wohngrundfläche auf bis zu 57 Quadratmeter. Der Außenbau dokumentiert demgegenüber den geringen Aufwand in der Planung und Ausgestaltung der Häuserzeilen. Über einem massiven Sockelgeschoss, das lediglich durch die Abfolge der Zugänge und Fenster unterteilt war, erhob sich ein Obergeschoss mit simpler Fachwerkgliederung (Abb. 4).

\section{Abb. 4: Essen, Arbeiterkolonie Westend, 1863, Häuserzeile aus} dem ersten Bauabschnitt

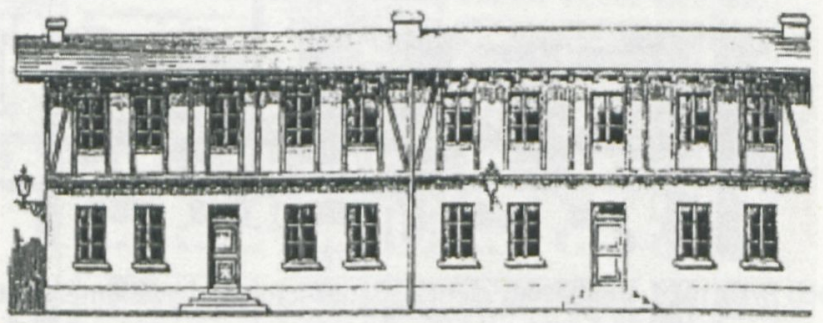

1871 entschloss sich die Firma, die Kolonie Westend zu erweitern. Fünf neue Häuserzeilen wurden zur bestehenden Bebauung in einer Doppelreihe quer angeordnet, wobei man den mittleren Bereich aussparte und bepflanzte, wodurch sich ein kleines begrüntes Zentrum für die Arbeiterkolonie ergab. Um deren Eigenständigkeit zu erhöhen, wurden zwei Versorgungseinrichtungen am südwestlichen Rand erbaut, eine so genannte „Konsumanstalt“, also ein Geschäft für die Waren des täglichen Gebrauchs, und eine Bierhalle.

96 Wohnungen wurden in diesem zweiten Bauabschnitt errichtet. Wiederum waren es entweder kleine Zweizimmerwohnungen mit einer Wohnküche oder aber eine etwas erweiterte Variante mit nunmehr drei Räumen (Abb. 5).

Die Häuserzeilen selbst waren gegenüber ihren Vorläuferbauten insgesamt größer dimensioniert, wobei das Dach zu einem eigenen Stockwerk ausgebaut und mittels großer Dachgauben eigenständig belichtet wurde. Wie schon im ersten Bauabschnitt war der Außenbau wenig gestaltet und nur durch die strenge Abfolge der Fensterachsen gegliedert. Anstelle des ursprünglich bevorzugten Fachwerkbaus favorisierte man nun einfache, vermutlich unverputzte Ziegelfronten, deren einziger architektonischer Anspruch in der Verwendung von Dreiecksgiebeln für die Dachgauben und von Segmentbögen für die Fensteröffnungen bestand. Ebenso anspruchslos wie die ursprüngliche Planung war demnach auch die zweite Bauphase in der Arbeiterkolonie Westend. 
Abb. 5: Essen, Arbeiterkolonie Westend, 1871, Häuserzeile aus dem zweiten Bauabschnitt

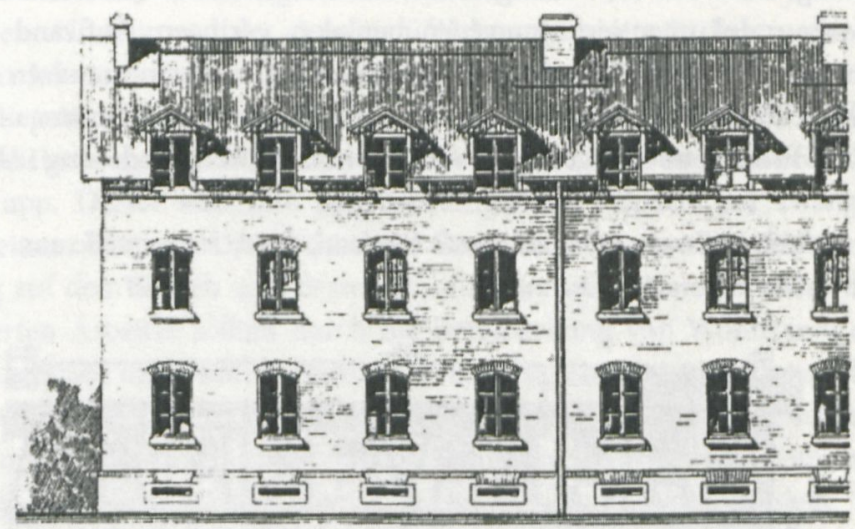

Dennoch war man in diesem neuen Bauabschnitt daran interessiert, den Außenraum deutlicher $\mathrm{zu}$ artikulieren. Was ursprünglich als eine reine Addition von Häuserreihen angelegt war, sollte nun zu einer kleinen, in sich geschlossenen Siedlungseinheit umgestaltet werden. Die neuen kollektiven Wohngebäude schlossen die Arbeiterkolonie zur anliegenden Straße ab, und durch die mittlere Freifläche entstand ein zentraler Platz, auf den die Wohnbebauung nun ausgerichtet war. Überdies boten die neuen Sekundäreinrichtungen der Arbeiterkolonie eine gewisse wirtschaftliche Teilautonomie. Für beide Bauabschnitte blieb aber der geometrisch streng angeordnete Zeilenbau prägend.

1872, und damit nur ein Jahr später als der zweite Bauabschnitt in der Kolonie Westend, wurde die Arbeiterkolonie Cronenberg am westlichen Rand der Gussstahlfabrik begonnen und 1874 fertiggestellt. In Bezug auf die räumliche Ausdehnung und den Umfang der für die Siedlung bereitgestellten Infrastruktur übertraf diese Kolonie alle übrigen Wohnbauunternehmungen der Firma Krupp, die Anfang der 1870er Jahre geplant und erbaut wurden. Das Bebauungsareal für Cronenberg umfasste allein 19 Hektar (Abb. 6). Errichtet wurden knapp 1.400 Wohnungen, wiederum in den schon vom zweiten Bauabschnitt der Kolonie Westend bekannten Varianten mit zwei respektive drei Räumen. Auch wurde der strenge Zeilenbau übernommen, allerdings nicht mehr in der monotonen Reihung, sondern nunmehr aufgelockert durch die Unterschiede in Größe, Lage und Ausrichtung der Häuserzeilen. Auf dem Raster eines rechtwinkligen Wegesystems mit Haupt- und Nebenstraßen wurden die Häuserzeilen gleichmäßig und vor allem mit einer gegenüber den anderen Arbeiterkolonien eher niedrigen Bebauungsdichte auf dem Areal verteilt. 
Abb. 6: Essen, Arbeiterkolonie Cronenberg, 1872-74, Grundriss der Gesamtanlage

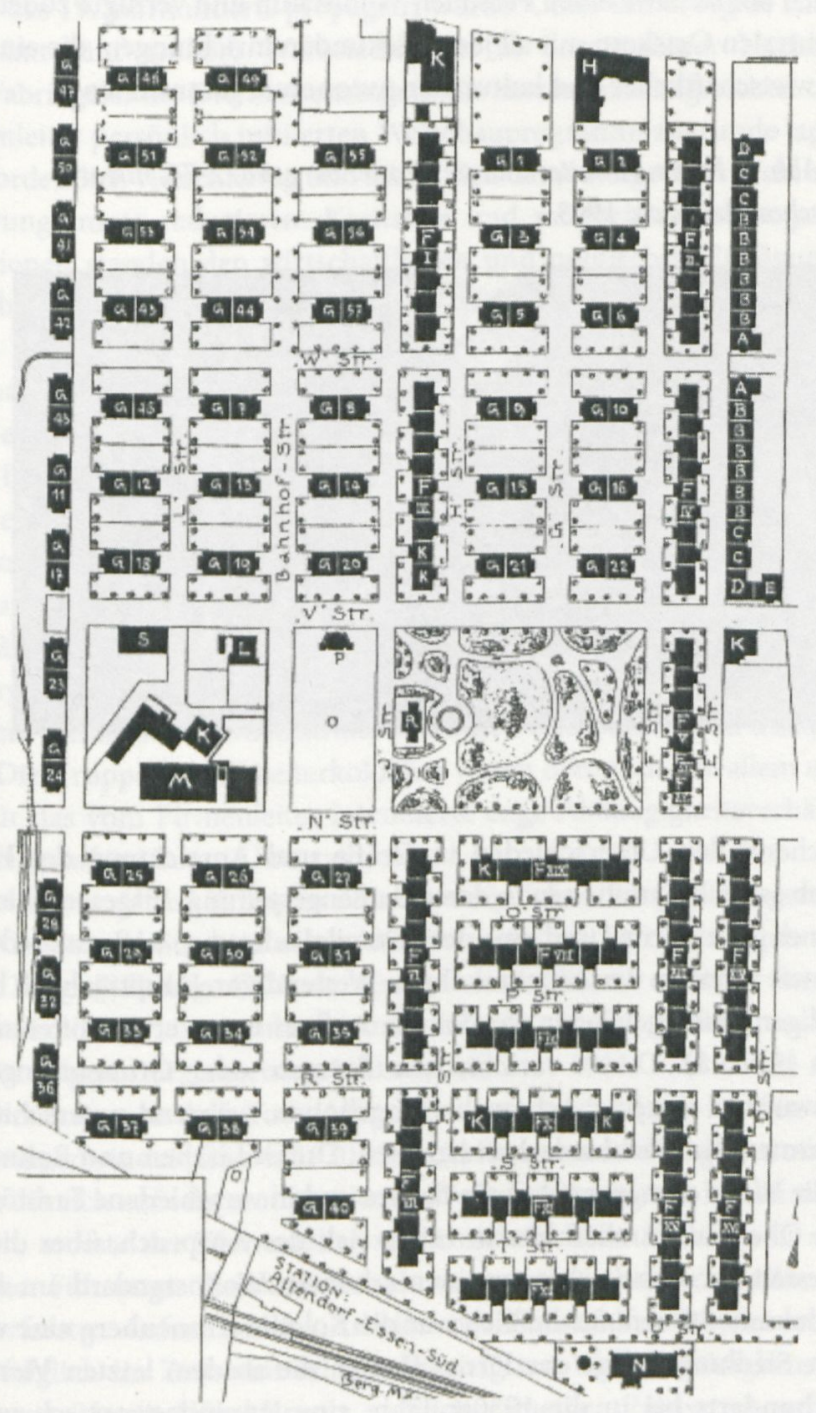

Zeitgenössische Photos illustrieren sehr gut, dass man auf die großflächige Bepflanzung mit Baumalleen und Vorgärten durchaus Wert legte (Abb. 7). Dementsprechend positionierte man im zentralen Bereich der Arbeiterkolonie einen Park mit organisch verlaufenden Spazierwegen. Diese Siedlungsmitte umfasste aber nicht nur einen begrünten Freiraum, sondern auch einen öffentlichen Marktplatz und die kollektiven Versorgungseinrichtungen, wie Konsumanstalt oder Magazinbau, und Gemeinschaftsgebäude, wie Bierhalle und Musikpavillon. Zwei Schulgebäude schlossen diese mittlere 
Achse zur östlichen Schmalseite ab. Was die Konzeption der Arbeiterkolonie Cronenberg somit entscheidend prägte, war ihre Analogie zu einer Kleinstadt, schließlich bot sie etwa 8.000 Personen Wohnraum und verfügte zudem über einen zentralen Ortskern mit all jenen Sekundäreinrichtungen, die ein hohes $\mathrm{Maß}$ an wirtschaftlicher und kultureller Autonomie garantierten.

Abb. 7: Essen, Arbeiterkolonie Cronenberg, 1872-74, historisches Photo, ca. 1903

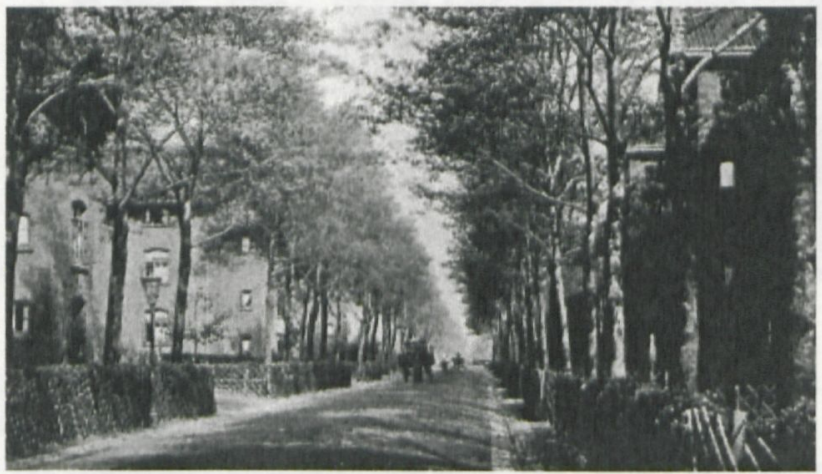

Entsprechend den Unterschieden in Größe und Ausrichtung der Häuserzeilen gab es auch Variationen in deren Außengestaltung. Insgesamt herrschte aber jener nüchterne und in der Detailgliederung äußerst reduzierte Habitus wie schon in der Arbeiterkolonie Westend vor. Hauptsächlich bei den mehrteiligen Zeilengebäuden wurden die Außenfronten etwas differenzierter gestaltet (Abb. 8). Durch verschiedene Formate oder Gruppierungen der Fenster wurden einzelne Achsen hervorgehoben, während unterschiedliche Giebelformen die Dachlandschaft belebten. Durch Lisenen und Rahmungen erhielt die Wand demgegenüber ein flaches und in verschiedene Farbtöne aufgeteiltes Oberflächenrelief. Hierin zeigte sich der Anspruch, über die reine Zweckgestalt der Arbeiterhäuser einen erhöhten Wohnstandard am Außenbau zu dokumentieren. Schließlich war die Kolonie Cronenberg eine weithin sichtbare Siedlungsanlage der Firma Krupp, die ab dem letzten Viertel des 19. Jahrhunderts bis in die 1930er Jahre eine zumindest optisch wichtige Dominante im Essener Stadtbereich darstellte.

Abb. 8: Essen, Arbeiterkolonie Cronenberg, 1872-74,

\section{Häuserzeile}

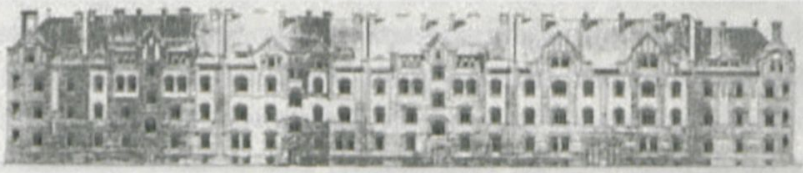


Die beiden untersuchten Arbeiterkolonien sind Musterbeispiele für einen paternalistischen Wohnungsbau, wie er unter Alfred Krupp in der zweiten Hälfte des 19. Jahrhunderts propagiert wurde. Unter seiner Ägide entstanden insgesamt fünf größere Werksiedlungen in unmittelbarer Nachbarschaft zum Fabrikgelände. Die Zielsetzungen, die diesem umfangreichen und vom Firmenleiter persönlich initiierten Wohnbauprogramm zugrunde lagen, sind außerordentlich vielschichtig und lassen sich demzufolge auch nicht auf einen Erklärungsansatz reduzieren. Karitative und möglicherweise sogar soziale Intentionen standen den wirtschaftlichen und politischen Erwägungen fast gleichberechtigt gegenüber. Trotz dieses breiten Spektrums von unterschiedlichen Motiven und Zielsetzungen ließ sich Alfred Krupp stets von einem pragmatischen Grundsatz leiten, der auf dem Wechselverhältnis von Leistung und Gegenleistung basierte: Wenn die Firma für ihre Arbeiter Wohnraum und einen bestimmten Lebensstandard in den Siedlungen zur Verfügung stellte, dann erwartete sie im Gegenzug deren unbedingte Bereitschaft, als loyale Lohnkräfte in den Produktionsbetrieben zu arbeiten. Zwar half der Arbeiterwohnungsbau der Firma Krupp, „die individuellen Wohnungsverhältnisse der Beschäftigten zu verbessern“, wie es Renate Kastorff-Viehmann (1981:153) hervorgehoben hat, „aber dennoch nicht unmittelbar mit dem Ziel, Wohlfahrt zu üben, sondern um Arbeitskräfte zu binden und politisch zu disziplinieren [...]. “Die Kruppschen Arbeiterkolonien waren demnach vor allem ein Sinnbild für das vom Firmenleiter intendierte enge Abhängigkeitsverhältnis der Arbeiterschaft zu ihrem Unternehmen.

Dabei war Alfred Krupp in seinen persönlichen Entscheidungen, was die Lage, Größe und Struktur seiner Arbeiterkolonien betraf, weitgehend autonom. Das großflächige Gelände seiner Gussstahlfabrik mitsamt dem für die Arbeiterkolonien vorgesehenen Außenbereich gehörte damals noch zu der eigenständigen Bürgermeisterei Altendorf. Erst 1901, und damit mehr als ein Jahrzehnt nach dem Tode Alfred Krupps, wurde dieses Dorf in die Stadt Essen eingemeindet (Günter 1970:134). Auf kommunale Vorgaben oder Richtlinien, die sich auf sein ambitioniertes Wohnbauprogramm hätten auswirken können, musste der Firmenleiter somit nicht achten. Zudem war sein persönlicher Führungsstil alles andere als kooperativ. In einem von ihm persönlich verfassten Aufruf an die „Arbeiter der Gußstahlfabrik“, der am 24. Juli 1872, folglich im Zeitraum seiner ehrgeizigsten Wohnbautätigkeit, erlassen wurde, versicherte Alfred Krupp, „daß ich in meinem Hause wie auf meinem Boden Herr sein und bleiben will“ (Baedeker 1912:101-102). Und noch 1887, in seinem Todesjahr, ließ er in einem Brief folgenden Leitsatz verlauten: „In unseren Colonien darf niemals ein Anderer einen Besitz haben und grundsätzlich sollten keine Anderen als Arbeiter der Fabrik (und Meister) dort wohnen“ (Berdrow 1928:427). Was in diesen zwei kurzen Sequenzen bereits unmissverständlich zum Ausdruck kommt, ist das außerordentlich stark ausgeprägte Selbstverständnis eines Fabrikbesitzers, der sich im traditionellen Sinne als Territorialherr mit beinahe schon uneingeschränkter Verfügungsund Entscheidungsgewalt verstand. Paternalismus war für Alfred Krupp also eine rein patriarchalische Handlungsstruktur, auf welche die Arbeiterschaft 
seiner Gussstahlfabrik wiederum pflichtbewusst reagierte oder reagieren musste, indem sie sich selbst in der Regel als „Kruppianer“ bezeichnete. Kaum deutlicher als in einem 1887 veröffentlichten Gedenkblatt zum Tode Alfred Krupps hätte man dieses paternalistische Abhängigkeitsprinzip formulieren können: „Ihr [das heißt die Kruppschen Arbeiter, Anmerkung des Verfassers] wußtet, was Ihr an Eurem Herrn hattet, und, als derselbe mit warnenden und mahnenden Worten zu Euch sprach, da fühltet Ihr Alle, daß nicht ein stolzer Besitzer zu Euch sprach, sondern wie ein Vater zu seinen Kindern redet, so klangen Euch seine Worte [...]“ (Sturm 1977:197).

Das hohe Maß an sozialer Reformtätigkeit, das sich in den zu Anfang erwähnten Siedlungskonzepten der englischen Unternehmer artikulierte, erreichte das Wohnbauprogramm von Alfred Krupp demnach nicht. Dennoch waren es genau diese englischen Modellvorstellungen, die ihn persönlich beeinflusst haben. Seit 1838/39 hatte Alfred Krupp mehrfach die britische Insel besucht, den gesamten Herbst und Winter 1871/72 im englischen Seebad Torquay/Devonshire verbracht und während dieser Aufenthalte eine Fülle von Anregungen für seine spätere Wohnbautätigkeit erhalten (Berdrow 1927:202-216, 1937:223-224; Klapheck 1930:11-17; KastorffViehmann 1981:154). Schon ein kurzer Vergleich zwischen der bereits genannten englischen Siedlung Saltaire von 1851 bis 1860 und der Kolonie Cronenberg von 1872 bis 1874 zeigt eine Vielzahl formaler Übereinstimmungen: Man muss diesbezüglich nur auf das orthogonale Straßenraster, den strengen Zeilenbau oder die unterschiedlichen Versorgungseinrichtungen verweisen, um die Analogien zu verdeutlichen. Zudem wurde in beiden Siedlungen ein großer Wert auf öffentliche Grünzonen gelegt, die vor allem der Erholung der Arbeiter und ihren Familien dienen sollten.

Folglich stellen die Wohnkolonien der Firma Krupp keine städtebaulichen Inventionen dar. Ihre Vorbilder waren jene englischen Arbeitersiedlungen, die vorwiegend von philanthropischen Unternehmern errichtet worden waren, um ihren Angestellten ein angemessenes Maß an Lebensqualität im Wohnumfeld zu garantieren. Das große Verdienst der Kruppschen Wohnbautätigkeit liegt eher darin, bestimmte Reformansprüche wie formale Merkmale dieser englischen Siedlungen in ihren eigenen Arbeiterkolonien konsequent verarbeitet zu haben, und dies über Jahrzehnte hinweg. Anlagen wie die Kolonie Cronenberg in Essen standen fortan für einen zwar aus England übernommenen, aber für deutsche Verhältnisse durchaus neuen Standard von werkseigenen Arbeitersiedlungen, die das Unternehmen über die firmeninternen Publikationen der Öffentlichkeit als so genannte „Wohlfahrtseinrichtungen" fortwährend präsentierte und dadurch werbewirksam vermarktete (Wohlfahrtseinrichtungen I-III 1902; Müller 1911; Baedeker 1912; Krupp 1912; Klapheck 1930). 


\section{Die Arbeiterkolonie der Textilfabrik Ulrich GMINDER IN REUTLINGEN}

Ähnlich wie bei der Gussstahlfabrik Krupp waren es auch bei der Textilfabrik Ulrich Gminder in Reutlingen zunächst wirtschaftliche Erwägungen, die zum Bau einer werkseigenen Arbeiterkolonie, dem so genannten "Gmindersdorf", führten (Howaldt 1982:329-360; Nerdinger 1988:114-118, 211-213; Reinborn 1996:87-88). Nach 1900 wuchs die Produktion in der Firma enorm an und erreichte 1903 ihren Höchststand. Aus dem Grunde entschloss sich die Firmenleitung noch im selben Jahr zur Errichtung einer Arbeitersiedlung in unmittelbarer Nähe zu den Fabrikanlagen, so dass der Erhalt des bereits vorhandenen Stammpersonals und die Gewinnung neuer Arbeitskräfte durch die Bereitstellung von Wohnraum gesichert werden sollten (Baer 1923:2); (Abb. 9).

Abb. 9: Reutlingen, Arbeitersiedlung Gmindersdorf, 1903-15, Grundriss der Gesamtanlage

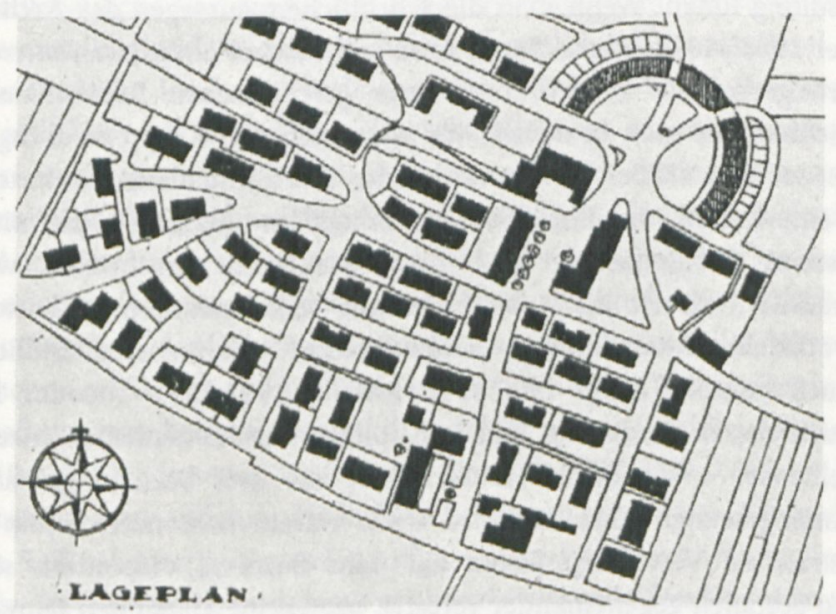

In Analogie zur Firma Krupp waren es auch bei der Textilfabrik Ulrich Gminder zuerst der Seniorchef des Unternehmens, Louis Gminder (18431904), und nach seinem Tod dessen Neffe und nachfolgender Firmenleiter, Emil Gminder (1873-1962), welche die Initiative zum Bau der Arbeiterkolonie persönlich einleiteten und deren Fertigstellung bis 1915 konsequent vorantrieben. Und schließlich betonte man auch in diesem Fall die karitativen Beweggründe der Firmenleiter, die zum Entschluss für die Errichtung einer Arbeiterkolonie geführt hatten. So wurde in einem Artikel zur Arbeitersiedlung von 1908 explizit hervorgehoben, „dass die soziale Frage vornehmlich eine Wohnungsfrage“ sei (Kuhn 1908:314; Baer 1923:3). Das Hauptmotiv für die Gründung dieser Arbeiterkolonie bestehe deshalb darin, „den Arbeitern [...] gesunde Wohnungen gegen billige Miete gewähren zu können“ 
(Kuhn 1908:314; Baer 1923:3). Eine mögliche Übereignung der Wohnungen an die Arbeiter und ihre Familien im Sinne kostengünstiger Ratenzahlungen oder dergleichen wurde jedoch von der Firmenleitung von vornherein ausgeschlossen: „Die Häuser bleiben im Besitz von Ulrich Gminder G.m.b.H. “ (Kuhn 1908:314; Baer 1923:3). Jene rigiden Vorstellungen von einem außerordentlich engen Abhängigkeitsverhältnis der Arbeiterschaft von ihrem Unternehmen, wie sie noch Alfred Krupp besaß, scheinen die Firmenleiter der Ulrich Gminder Textilfabrik allerdings nicht vertreten zu haben. Dennoch galt auch in dieser Arbeitersiedlung das Gebot, „dass deren Bewohner in zufriedenem Sinne nach den Fabrikkaminen hinüberschauen, deren Rauch für sie menschliches Glück bedeutet, da er identisch ist mit Arbeit, Lohn und Nahrung“ (Kuhn 1908:314).

FürdiePlanungderSiedlungwurdederMünchnerReformarchitektTheodor Fischer beauftragt, der bis 1908 insgesamt 48 Wohngebäude in 19 verschiedenen Gebäudetypen mit etwa 150 Arbeiterwohnungen zwischen 56 und 92 Quadratmetern, die meisten davon mit zwei oder drei Räumen, erbaute. 1915 fügte er noch ein Altenheim für die pensionierten Arbeiter am nördlichen Rand der Siedlung hinzu. Wenn man die schriftlichen Aussagen des Architekten (Fischer 1908:313) berücksichtigt, dann war Fischer eher daran interessiert, die Arbeiterkolonie in der Form einer geschlossenen Reihenhausanlage zu errichten, wie man es damals vor allem von den Krupp-Siedlungen aus den 1860er und 1870er Jahren her kannte. Die Firmenleiter forderten hingegen eine Kolonie aus Einzelhäusern, erbaut im offenen System mit angeschlossenen Gartenflächen. Was ihnen wiederum vorschwebte, war ein "Gartendorf", wie sie die Arbeitersiedlung selbst nannten und wie es in deren offiziellen Bezeichnung - „Gmindersdorf " - unmissverständlich zum Ausdruck kommt (Kuhn 1908:314; Howaldt 1982:333). Theodor Fischer entsprach dieser Forderung und konzipierte unterschiedliche Einzel- und Doppelhäuser sowie kurze Häuserreihen aus drei beziehungsweise vier zusammengesetzten Einzelhäusern. Diese waren nicht nach einem streng geometrischen Verteilungssystem auf dem Bauareal disponiert, sondern wurden gestaffelt gegeneinander versetzt, um „keine klaffenden Durchblicke zu[zu]lassen“, wie es der Architekt (Fischer 1908:313) selbst bezeichnete. Dadurch entstand der Eindruck einer organisch gewachsenen Dorfanlage, der durch das leicht geschwungene Straßennetz noch verstärkt wurde. Im nördlichen Bereich gestaltete der Architekt einen kleinen Marktplatz mit den notwendigen Versorgungs- und Sekundäreinrichtungen. Vervollständigt wurde die Gesamtanlage durch das Altenheim, das mit seiner halbkreisförmigen Außenkontur und seinem repräsentativen Mitteltrakt das Siedlungsareal an der nördlichen Anhöhe auch heute noch optisch begrenzt. Bei der Planung der Wohnhäuser legte Fischer demgegenüber größtmöglichen Wert auf die Variation in der Außengestaltung. Zum Teil ließ er sich dabei von der örtlichen Bautradition schwäbischer Land- und Bauernhäuser beeinflussen (Abb. $10)$. 
Abb. 10: Reutlingen, Arbeitersiedlung Gmindersdorf, 1903-15, Einzelhäuser

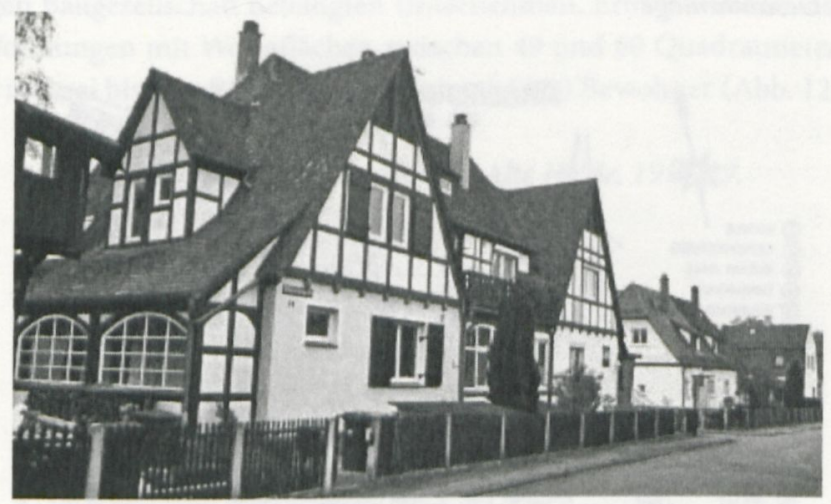

Die städtebauliche Vorlage für die Arbeitersiedlung Gmindersdorf stammte dagegen aus England. Wie schon Alfred Krupp besuchte auch Emil Gminder die britische Insel und hielt sich dort 1893 für einen längeren Zeitraum auf (Howaldt 1982:334). Wahrscheinlich hat er währenddessen die damals modernsten englischen Arbeitersiedlungen kennengelernt. Seine präzisen Vorstellungen hinsichtlich der Planung von Gmindersdorf scheinen diese Annahme jedenfalls zu bestätigen. Man braucht nur die Gesamtanlage von Gmindersdorf dem Organisationsschema der englischen Arbeitersiedlung Bournville in der Nähe von Birmingham gegenüberzustellen, die von den Unternehmern George und Richard Cadbury ab 1887 in unmittelbarer Nachbarschaft zu ihrer Schokoladenfabrik errichtet wurde (Weißbach/Mackowsky 1910:123-126; Posener 1968:29-35; Howaldt 1982:334); (Abb. 11). Vergleichbar ist unter anderem die lockere Bebauung mit Einzel-, Doppel- oder kurzen Reihenhäusern, die weiträumige Anlage von Gartenflächen und die teilweise geschwungene und damit organisch wirkende Straßenführung. Wie schon bei den früheren Krupp-Siedlungen sind auch bei der Planung für die Arbeiterkolonie der Textilfabrik Ulrich Gminder die modernsten Tendenzen des englischen Siedlungsbaus vom Ende des 19. Jahrhunderts eingeflossen. Schon 1908 bezeichnete J. Altenrath $(1908: 24,27)$ die Arbeitersiedlung Gmindersdorf dementsprechend als "neueste und vielleicht vollendetste Arbeiterkolonie" und umschrieb deren Bedeutung am Ende seines Artikels mit folgenden Worten: „Arbeitgeber und Künstler haben sich hier ein bleibendes Denkmal gesetzt." Zwei Jahre später betonten Karl Weißbach und Walter Mackowsky (1910:273) in ihrer bekannten Schrift über die historische Entwicklung des Arbeiterwohnhauses, dass die Kolonie Gmindersdorf „in künstlerischer Beziehung unter die besten derartigen Schöpfungen zu rechnen ist" und diese deshalb „den berühmten Kruppschen und englischen Kolonien ebenbürtig an die Seite gestellt werden muß." 
Abb. 11: Bournville bei Birmingham, Arbeitersiedlung der Schokoladenfabrik Cadbury, ab 1887, Grundriss der Gesamtanlage

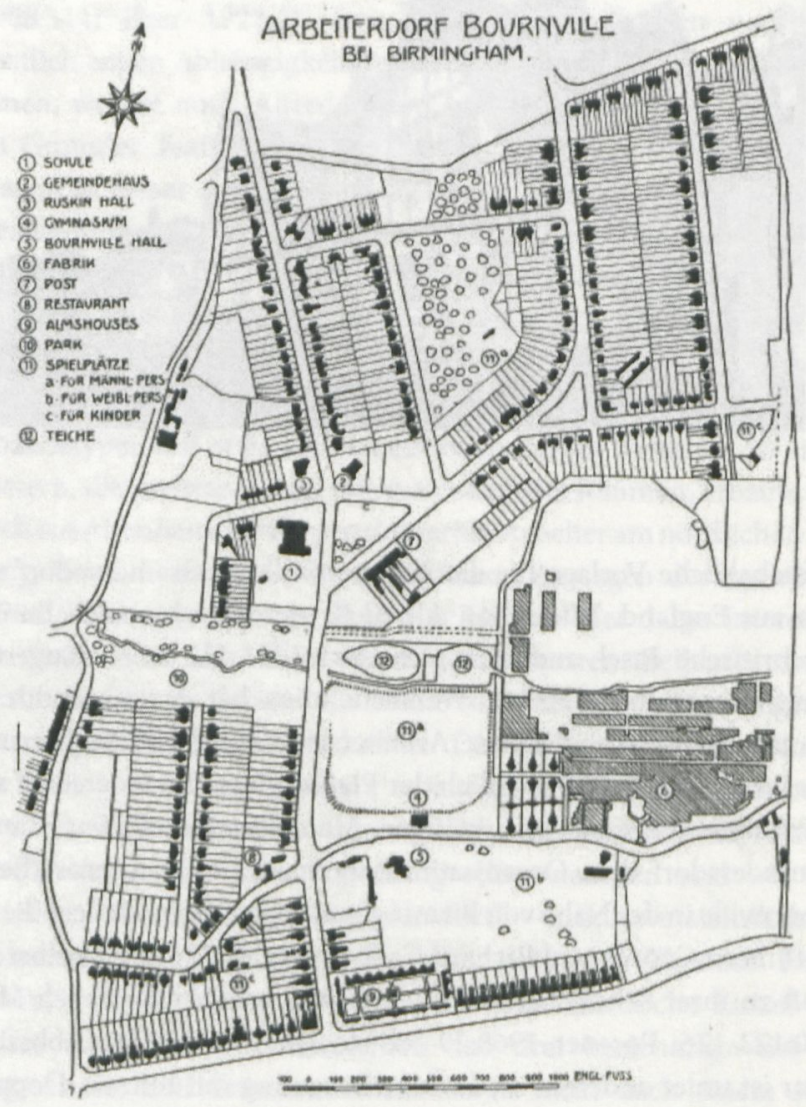

\section{Die Arbeitersiedlung Alte Heide in München}

Eine weitere Arbeiterkolonie von Theodor Fischer ist die Siedlung Alte Heide in München von 1918 bis 1929 (Gut 1919:410-413; Nerdinger 1988:121-123, 286-288; Reinborn 1996:88). Der Bauträger war zwar eine "Gemeinnützige Baugesellschaft", doch wurde diese Ende August 1918 von mehreren bekannten Münchner Unternehmen gegründet, darunter die Bayerische Motorenwerke AG, die Löwenbräu AG, die Lokomotivfabrik Maffei und die Bayerischen Geschützwerke München Krupp (Krause 1991:260-263; Lutzenberger 2004:25). Ihr primärer Zweck bestand in der Schaffung von Wohnraum für die Arbeiter dieser Unternehmen, so dass die Siedlung Alte Heide bereits während ihrer Errichtung als so genannte „erste Werksiedlung der Münchner Industrie“ (Krause 1991:261) bekannt wurde. Ihre Lage 
im nördlichen Randbereich der Stadt entsprach einer räumlich günstigen Anbindung an die Betriebs- und Produktionsstätten jener an der Gemeinnützigen Baugesellschaft beteiligten Unternehmen. Erbaut wurden insgesamt 786 Wohnungen mit Wohnflächen zwischen 49 und 60 Quadratmetern, aufgeteilt in zwei bis vier Räumen, für maximal 4.000 Bewohner (Abb. 12).

Abb. 12: München, Arbeitersiedlung Alte Heide, 1918-29,

Grundriss der Gesamtanlage

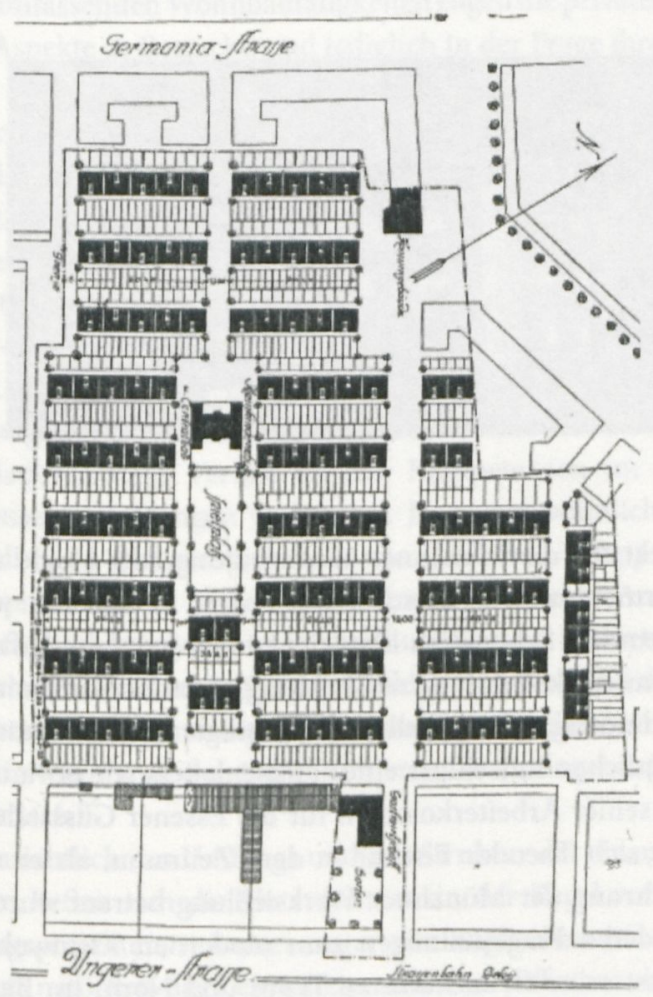

Das Anlageschema ist mit den bereits genannten Krupp-Siedlungen durchaus zu vergleichen, was nicht weiter erstaunt, wurden doch bei dem übergeordneten Bebauungsplan externe Anregungen verarbeitet, die von dem damaligen Leiter des Kruppschen Baubüros, dem Regierungsbaumeister Robert Schmohl, stammten (Gut 1919:411). So wählte Fischer als zugrunde liegenden Wohnbautypus den einfachen Zeilenbau, der nunmehr streng parallel angeordnet, auf dem gerasterten Areal gleichmäßig verteilt ist. Die Freiflächen zwischen den Häuserzeilen wurden den Bewohnern als Kleingärten zur Verfügung gestellt, während ein breiter Grünstreifen entlang der mittleren Hauptachse öffentlich genutzt werden konnte. Für den Gemeinschaftsbedarf wurden noch mehrere Sekundäreinrichtungen, wie Kindergarten oder Badeanstalt, auf dem Siedlungsareal verteilt. 
Ebenso nüchtern wie das Anlageschema ist auch die Außengestaltung der Häuserzeilen; im Grunde handelt es sich um einfache Putzfassaden, deren Detailgliederung durch den Rhythmus unterschiedlich gestalteter Fensterachsen differenziert wird. Die Betonung der Mitte wie der Flanken ist das Resultat, wodurch der Eindruck monotoner Achsenreihung verhindert werden sollte (Abb. 13).

Abb. 13: München, Arbeitersiedlung Alte Heide, 1918-29, Häuserzeilen

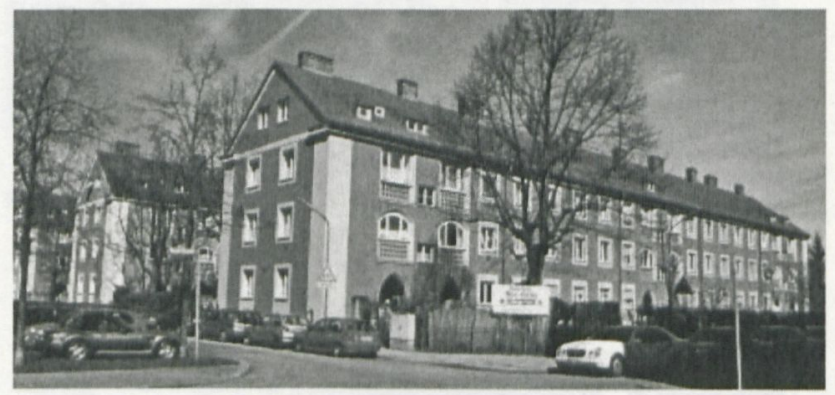

Dass bei dem Projekt für die Münchner Werksiedlung Alte Heide spezielle Vorstellungen firmeninterner Entscheidungsträger verarbeitet wurden, ist eher unwahrscheinlich. Schließlich handelte es sich bei dem Auftrag zur Siedlungsplanung um die kollektive Entscheidung eines Konsortiums mehrerer, an der Gemeinnützigen Baugesellschaft beteiligter Unternehmen, und nicht um den persönlichen Entschluss eines Firmenleiters wie etwa im Falle Alfred Krupps und seiner Arbeiterkolonien für die Essener Gussstahlfabrik. Zudem beschäftigte sich Theodor Fischer in dem Zeitraum, als er mit der Planung und Ausführung der Münchner Werksiedlung betraut wurde, vorwiegend mit besonderen Fragestellungen zum modernen Kleinwohnungsbau, woraus zunächst seine Grundsätze zu Typus und Norm im Bauwesen und daran anschließend seine theoretischen Vorstellungen zum städtischen Wohnungsbau resultierten (Fischer 1919:50, 1922:30-39). Fischer scheint somit präzise Vorstellungen sowohl von der zugrunde liegenden Bebauungsstruktur und der Gesamterscheinung der Werksiedlung als auch von der Grundrissdisposition und Außengestalt der einzelnen Wohnzeilen gehabt zu haben. Demzufolge mussten weder die Unternehmen, noch die Baugesellschaft eigene Vorstellungen diesbezüglich formulieren, sondern konnten vielmehr alle fachspezifischen Entscheidungen einem in dieser Hinsicht außerordentlich kompetenten Architekten überlassen. Schon 1919 bezeichnete der Münchner Stadtbaudirektor Albert Gut (1919:411) die Siedlung Alte Heide dementsprechend als „ein in sich geschlossenes Spiegelbild innerer Wahrheit.“ 


\section{DEutsche Arbeiterkolonien VON DER ZWEITEN HÄLFTE DES 19. BIS IN DIE ZWANZIGER JAHRE DES 20. JAHRHUNDERTS}

Wenn man die genannten deutschen Arbeiterkolonien im Gesamten betrachtet, ergeben sich in vielerlei Hinsicht Gemeinsamkeiten: Zunächst einmal waren es keineswegs nur wirtschaftliche oder ausschließlich karitative Erwägungen, die zur Errichtung dieser Siedlungen führten. Für ihre teilweise umfassenden Wohnbautätigkeiten zogen die privaten Unternehmen stets beide Aspekte in Betracht, und lediglich in der Frage ihres jeweiligen Stellenwertes gab es graduelle Unterschiede. Damit verbunden war eine meist geschickt kalkulierte Außenwirkung jener Siedlungsmaßnahmen, die der Öffentlichkeit als Einrichtungen privater Wohlfahrt präsentiert wurden, um die sozialen Ambitionen des jeweiligen Unternehmens ausdrücklich hervorzuheben. Paternalistische Grundsätze blieben dennoch wirksam, schließlich weigerten sich die Unternehmen konsequent, ihren Arbeitern in irgendeiner Weise - und sei es in genossenschaftlicher Form - die Wohnungen zu übertragen. So wurde erst 1916 eine Siedlungsgenossenschaft der Kruppschen Werksangehörigen in Essen gegründet (Schlandt 1970:98). Vermutlich wollte man dadurch dem Verlust eigener Kompetenzen im Sinne freier Unternehmensentscheidungen vorbeugen. Jene ungewöhnlich stark ausgeprägten Kontroll- und Befugnisgewalten, wie sie Alfred Krupp für sich persönlich in Anspruch nahm, stellen aber ohne Zweifel eine Ausnahme dar. Derart patriarchalisch ausgerichtete Handlungsweisen können lediglich bei dem Firmenleiter der Essener Gussstahlfabrik und nicht bei den anderen genannten Unternehmern nachgewiesen werden. In dieser Hinsicht war Alfred Krupp mit seinem äußerst ambitionierten Wohnungsbauprogramm sicherlich ein Sonderfall.

Hinsichtlich des Umfangs und der Struktur der einzelnen Siedlungsmaßnahmen ließen sich die Unternehmen in der Regel von bereits realisierten Siedlungsprojekten, mitunter sogar von englischen Modellen, beeinflussen. Hier galt natürlich das Leitprinzip moderner Effizienz, konnte man doch an diesen schon bestehenden Siedlungsanlagen deren tatsächliche Leistungsfähigkeit überprüfen. Die Bereitstellung von Sekundäreinrichtungen sollte überdies ein hohes Maß an Siedlungsautonomie garantieren, so dass einige Arbeiterkolonien beinahe schon den Status von Dörfern oder Kleinstädten innerhalb des teilweise urbanen Umfeldes erlangten.

Und letztlich gab es auch eine Vielzahl formaler Analogien: Nicht selten wurde der strenge Zeilenbau gewählt. Den Außenraum gestaltete man häufig als begrünte Freifläche, die sowohl der Erholung als auch der privaten Selbstversorgung dienen konnte. Die Wohngrundrisse waren zumeist auf wenige standardisierte Typen reduziert. Bei den größeren Arbeitersiedlungen wurde die Außengestaltung vorwiegend durch ein äußerst begrenztes Formenrepertoire bestimmt, das Variationen oder reichere Detailgliederungen nur in beschränktem Maße zuließ. Hier offenbarte sich ein Purismus in der architektonischen Formgebung, der nicht nur von wirtschaftlichen Erwägungen 
getragen wurde. Die Bauaufgabe Arbeiterkolonie verlangte zumindest in der damaligen Zeit nach nüchternen und vor allem reduzierten Lösungsmustern.

Nach dem Ende des Ersten Weltkriegs fanden viele Gestaltungsprinzipien, die bei der Planung dieser Arbeiterkolonien erarbeitet worden waren, Eingang in den kommunalen Siedlungs- und Wohnungsbau der Weimarer Republik. Man muss diesbezüglich nur auf die Siedlung Westhausen in Frankfurt verweisen, die von Ernst May zwischen 1929 und 1931 errichtet wurde (Reinborn 1996:38, 107-108); (Abb. 14). In ihrer strengen Zeilenbauweise mit der teilweise unterschiedlichen Ausrichtung der Häuserzeilen vor allem im Randbereich der Siedlung ähnelt sie dem Anlageschema der Kruppschen Arbeiterkolonie Cronenberg in Essen von 1872 bis 1874. Die von Bruno Taut zwischen 1925 und 1927 errichtete Großsiedlung Britz in Berlin wurde demgegenüber von der Arbeitersiedlung Gmindersdorf beeinflusst, was nicht weiter erstaunlich ist, war doch Taut von 1904 bis 1908 in Theodor Fischers Architekturbüro angestellt gewesen und hatte bei der Planung für die Gmindersdorfer Anlage mitgearbeitet (Howaldt 1982:333; Nerdinger 1988:118; Reinborn 1996:115116). Der halbkreisförmige Baukomplex des Altenheims, den Fischer als nördlichen Abschluss der Gmindersdorfer Siedlung konzipierte, wurde von Taut bei seinem Entwurf für den zentralen hufeisenförmigen Wohnring in seiner Berliner Siedlung offenkundig verarbeitet (Abb. 15). Diese strukturellen wie formalen Analogien zeigen wiederum, dass die deutschen Arbeiterkolonien ab der zweiten Hälfte des 19. Jahrhunderts einen außerordentlich wichtigen Beitrag für die weitere Entwicklung der modernen Stadt- und Siedlungsplanung im 20. Jahrhundert geleistet haben. Vor allem aber trugen sie, und dies hat Walter Kieß (1991:392) ausdrücklich hervorgehoben, „zu einem Zeitpunkt, als es noch keine geordnete Siedlungstätigkeit der Städte gab, im Rahmen einer urbanen Dezentralisation zur Verbesserung der Lebensqualität und des persönlichen Lebenszuschnitts der Arbeiter bei.“

Abb. 14: Frankfurt, Siedlung Westhausen, 1929-31, Grundriss der Gesamtanlage

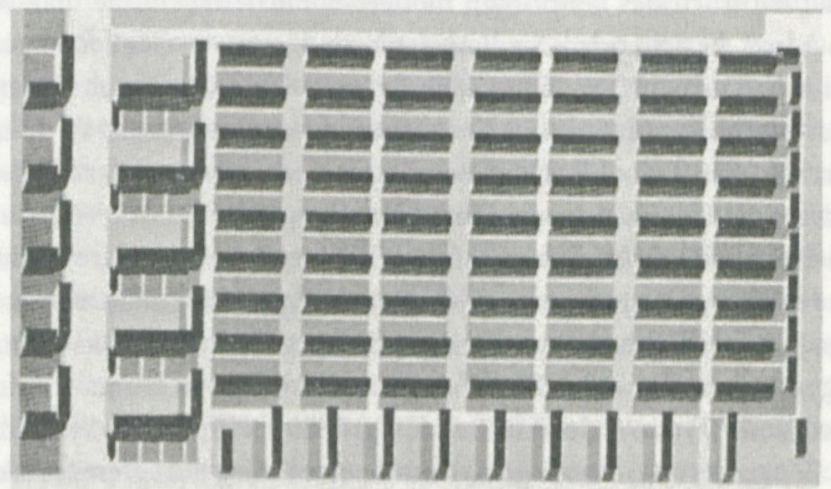


Abb. 15: Berlin, Großsiedlung Britz, 1925-27, Grundriss der Gesamtanlage

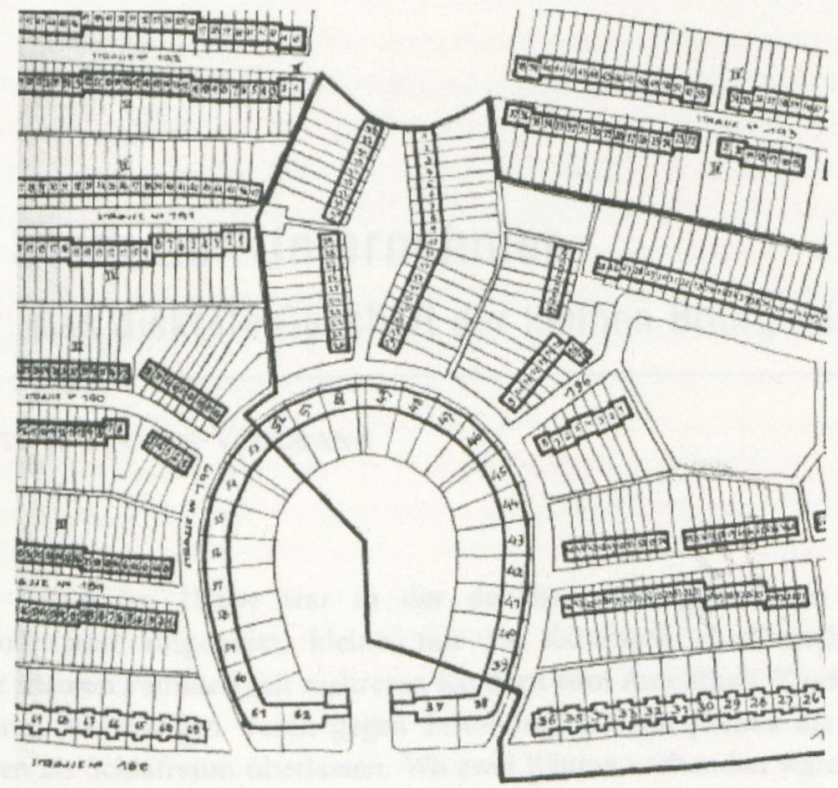




\section{DEUTSCHE UNTERNEHMER UND IHRE ARBEITERKOLONIEN IM 19. UND FRÜHEN 20. JAHRHUNDERT}

\section{Literatur}

Altenrath, J. (1908): Die Arbeiterkolonie „Gmindersdorf“ der Firma Ulrich Gminder in Reutlingen. In: Zeitschrift für Wohnungswesen VII (2), 2327

Baedeker, D. (1912): Alfred Krupp und die Entwicklung der Gußstahlfabrik zu Essen. Essen: Verlagshandlung G. D. Baedeker

Baer, C. H. (1923): Kleinbauten und Siedelungen. Stuttgart: Verlag Julius Hoffmann

Berdrow, W. (1927): Alfred Krupp. Berlin: Verlag Reimar Hobbing

Berdrow, W. (1928): Alfred Krupps Briefe 1826-1887. Berlin: Verlag Reimar Hobbing

Berdrow, W. (1937): Alfred Krupp und sein Geschlecht. Geschichte eines deutschen Familienunternehmens. Berlin: Verlag für Sozialpolitik, Wirtschaft und Statistik

Dreysse, D. W. (1988): Ernst May. Housing Estates. Architectural Guide to eight new Frankfort Estates 1926-1930. Frankfurt/M.: Verlag Dieter Frikke

Essen und Umgebung (1938): Grieben Reiseführer. Bd. 39. Berlin: GriebenVerlag

Fischer, T. (1908): Gmindersdorf. Arbeiterkolonie von Ulrich Gminder GmbH in Reutlingen. In: Moderne Bauformen VII (8), 313

Fischer, T. (1919): Wie stellt sich der Architekt zur Normalisierung und Typisierung? In: Süddeutsche Bauzeitung 29, 15. Juni 12, 50

Fischer, T. (1922): Sechs Vorträge über Stadtbaukunst. München/Berlin: Oldenbourg Verlag

Günter, R. (1970): Krupp und Essen. In: Warnke, M. (Hrsg.): Das Kunstwerk zwischen Wissenschaft und Weltanschauung. Gütersloh: Bertelsmann Kunstverlag, 128-174

Gut, A. (1919): Zwei neue Kleinwohnungsanlagen in München. In: Zentralblatt der Bauverwaltung 39 (68/69), 405-407, 410-413

Howaldt, G. (1982): Die Arbeitersiedlung Gmindersdorf in Reutlingen. In: Mai, E. / Pohl, H. / Waetzoldt, S. (Hrsg.): Kunstpolitik und Kunstförderung im Kaiserreich. Kunst im Wandel der Sozial- und Wirtschaftsgeschichte. Berlin: Gebr. Mann Verlag, 329-360

Kastorff-Viehmann, R. (1981): Wohnungsbau für Arbeiter. Das Beispiel Ruhrgebiet bis 1914. Aachen: Klenkes Verlag

Kieß, W. (1991): Urbanismus im Industriezeitalter. Von der klassizistischen Stadt zur Garden City. Berlin: Verlag Ernst \& Sohn

Klapheck, R. (1930): Siedlungswerk Krupp. Berlin: Ernst Wasmuth Verlag Krause, L. (1991): Münchner Geschossiedlungen der 50er Jahre. Ein Forschungsbeitrag zum Wohnungsbau in der Bundesrepublik Deutschland. Dissertationen zur Bayerischen Landes- und Münchner Stadtgeschichte. 
Neue Schriftenreihe des Stadtarchivs München. München: Kommissionsverlag UNI-Druck München

Krupp (1912): Krupp 1812-1912. Zum 100jährigen Bestehen der Firma Krupp und der Gussstahlfabrik zu Essen. Jena: Verlag Gustav Fischer

Kuhn, W. (1908): Gmindersdorf. Arbeiterkolonie von Ulrich Gminder GmbH in Reutlingen. In: Moderne Bauformen VII (8), 314

Lutzenberger, K. (ca. 2004): Alte Heide. Geschichte und Geschichten. Bd. I. Von der Schafweide zur Arbeitersiedlung. München: Selbstverlag Kulturverein Alte Heide

Müller, C. G. (1911): Krupp’s Gussstahlfabrik. Düsseldorf: Verlag August Bagel

Nerdinger, W. (1988): Theodor Fischer. Architekt und Städtebauer. Ausstellung der Architektursammlung der Technischen Universität München und des Münchner Stadtmuseums in Verbindung mit dem Württembergischen Kunstverein. Berlin: Verlag Ernst \& Sohn

Posener, J. (Hrsg.) (1968): Ebenezer Howard. Gartenstädte von morgen. Das Buch und seine Geschichte. Berlin/Frankfurt/M./Wien: Ullstein Verlag

Reinborn, D. (1996): Städtebau im 19. und 20. Jahrhundert. Stuttgart/Berlin/ Köln: Verlag W. Kohlhammer

Schlandt, J. (1970): Die Kruppsiedlungen - Wohnungsbau im Interesse eines Industriekonzerns. In: Helms, H. G. / Janssen, J. (Hrsg.): Kapitalistischer Städtebau. Analysen von Lucius Burckhardt, Hans G. Helms, Jörn Janssen u. a. Neuwied/Berlin: Hermann Luchterhand Verlag, 95-111

Sturm, H. (1977): Fabrikarchitektur Villa Arbeitersiedlung. München: Heinz Moos Verlag

Walther, I. (Hrsg.) (1979): Paris-Berlin 1900-1933. Übereinstimmungen und Gegensätze Frankreich-Deutschland. Ausstellung im Centre National d'Art et de Culture Georges Pompidou, München: Prestel Verlag

Weißbach, K. / Mackowsky, W. (1910): Das Arbeiterwohnhaus. Berlin: Ernst Wasmuth Verlag

Wohlfahrtseinrichtungen I-III (1902): Wohlfahrtseinrichtungen der Gussstahlfabrik von Friedrich Krupp zu Essen a. d. Ruhr. 3 Auflage, Essen: Buchdruckerei der Gussstahlfabrik Friedrich Krupp. (1. Aufl. 1876)

\section{Abbildungen}

Abb. 1: Saltaire bei Bradford, Arbeitersiedlung der Textilfabrik, ab 1851, Grundriss der Gesamtanlage; Posener (1968:25)

Abb. 2: Essen, Arbeiterkolonien der Gussstahlfabrik Krupp, 1902, Übersichtskarte; Wohlfahrtseinrichtungen II (1902:XIV)

Abb. 3: Essen, Arbeiterkolonie Westend, 1863/1871, Grundriss der Gesamtanlage; Wohlfahrtseinrichtungen II (1902:1)

Abb. 4: Essen, Arbeiterkolonie Westend, 1863, Häuserzeile aus dem ersten Bauabschnitt; Wohlfahrtseinrichtungen II (1902:2)

Abb. 5: Essen, Arbeiterkolonie Westend, 1871, Häuserzeile aus dem zweiten 
Abb. 6: Essen, Arbeiterkolonie Cronenberg, 1872-74, Grundriss der Gesamtanlage; Wohlfahrtseinrichtungen II (1902:17)

Abb. 7: Essen, Arbeiterkolonie Cronenberg, 1872-74, historisches Photo, ca. 1903; Baedeker (1912:105)

Abb. 8: Essen, Arbeiterkolonie Cronenberg, 1872-74, Häuserzeile; Wohlfahrtseinrichtungen II (1902:18-19)

Abb. 9: Reutlingen, Arbeitersiedlung Gmindersdorf, 1903-15, Grundriss der Gesamtanlage; Nerdinger (1988:211)

Abb. 10: Reutlingen, Arbeitersiedlung Gmindersdorf, 1903-15, Einzelhäuser; eigenes Foto

Abb. 11: Bournville bei Birmingham, Arbeitersiedlung der Schokoladenfabrik Cadbury, ab 1887, Grundriss der Gesamtanlage; Weißbach/Mackowsky (1910:122)

Abb. 12: München, Arbeitersiedlung Alte Heide, 1918-29, Grundriss der Gesamtanlage; Gut (1919:411)

Abb. 13: München, Arbeitersiedlung Alte Heide, 1918-29, Häuserzeilen; eigenes Foto

Abb. 14: Frankfurt, Siedlung Westhausen, 1929-31, Grundriss der Gesamtanlage; Dreysse (1988:19)

Abb. 15: Berlin, Großsiedlung Britz, 1925-27, Grundriss der Gesamtanlage; Walther (1979:391) 